\title{
Performance and Microbial Community Analysis of an Electrobiofilm Reactor for Chelated-NO Removal Enhanced by Ferrous-EDTA
}

\author{
Nan Liu ${ }^{1}$, yingying $\mathrm{Li}^{1}$, Dujuan Ouyang ${ }^{1}$, Changyong Zou ${ }^{1}$, Wei Li ${ }^{2}$, Jihong Zhao ${ }^{3}$, jixiang \\ $\mathrm{li}^{4}$, wenjuan wang ${ }^{4}$, and Jiajun $\mathrm{Hu}^{5}$ \\ ${ }^{1}$ Zhengzhou University of Light Industry \\ ${ }^{2}$ Zhejiang University \\ ${ }^{3}$ Henan Radio and TV University \\ ${ }^{4}$ Chinese Academy of Sciences \\ ${ }^{5}$ Shanghai University
}

June 19, 2020

\begin{abstract}
The biological reduction of ferrous ethylenediaminetetraacetic acid (EDTA-FeII-NO and EDTA-FeIII) is an important process in integrated electrobiofilm reduction method, and this method has been regarded as a promising alternative for removing NOx from industrial boiler flue gas. EDTA-FeII-NO and EDTA-FeIII are crucial substrates that should be biologically reduced at a high rate. However, they would inhibit one another's reduction processes when they are present together, which might limited the further promotion of this integrated method. In this study, an integrated electrobiofilm reduction system with high reduction rate of EDTA-FeII- NO and EDTA-FeIII was established. The microbial communities in electrobiofilms were mainly studied to analyze their changes during the reduction of these two substrates under different conditions. It presents a better performance of substrates- resistance shock loading and high microbial diversities compared with the conventional chemical absorption-biological reduction system. High-throughput sequencing analysis showed that the changes in concentrations of EDTA-FeII-NO and EDTA-FeIII significantly impacted the genera of the microbial community. Alicycliphilus, Enterobacteriaceae and Raoultella were found to be the dominant genera ( $>25 \%$, respectively) involved in EDTA-FeII-NO reduction. As an EDTA-FeIII reducing bacteria, Chryseobacterium can endure shock loading of substrates. Ochrobactrum can reduce nitrate using electrons and exhibited better stability under shock loading. Furthermore, higher microbial diversity and stable reactor operation could be achieved when the concentrations of EDTA-FeII-NO and EDTA-FeIII approached the same value.
\end{abstract}

\section{Hosted file}

2020.05.31 original manuscript.docx available at https://authorea.com/users/334916/articles/ 460869-performance-and-microbial-community-analysis-of-an-electrobiofilm-reactor-forchelated-no-removal-enhanced-by-ferrous-edta 